\title{
Saúde do trabalhador: um olhar para o Centro Referencial de Saúde
}

\author{
Occupational health: a look at the Reference Health Center \\ Salud laboral: una mirada al Centro de Salud de Referencia
}

Recebido: 05/03/2021 | Revisado: 12/03/2021 | Aceito: 18/03/2021 | Publicado: 23/03/2021

Jennefer Emily Maraia Soares
ORCID: https://orcid.org/0000-0002-2249-2363
E-mail: jennefer.emily@unemat.br
Universidade do Estado de Mato Grosso Carlos Alberto Reyes Maldonado, Brasil
Julliana Ferrari Campêlo Libório de Santana
ORCID: https://orcid.org/0000-0002-7773-3398
E-mail: julliana.ferrari@unemat.br
Rosane Maria Andrade Vasconcelos
ORCID: https://orcid.org/0000-003-4746-1448
Universidade do Estado de Mato Grosso Carlos Alberto Reyes Maldonado, Brasil
E-mail: rosane@unemat.br
Paola Souza Santos
Universidade do Estado de Mato Grosso Carlos Alberto Reyes Maldonado, Brasil
ORCID: https://orcid.org/0000-0002-5419-4862
E-mail: pa.s.santos@hotmail.com
Mateus Vital Silva Rocha
Universidade do Estado de Mato Grosso Carlos Alberto Reyes Maldonado, Brasil
ORCID: https://orcid.org/0000-0003-4075-8142
E-mail: mateus.vital@unemat.br
Thays Andrade Apolinário
Universidade do Estado de Mato Grosso Carlos Alberto Reyes Maldonado, Brasil
ORCID: https://orcid.org/0000-0003-1695-2092
E-mail: thays_apolinario@hotmail.com

\section{Resumo}

A Saúde do Trabalhador caracteriza-se como um campo de práticas e de conhecimentos estratégicos voltados para analisar e intervir nas relações de trabalho que provocam doenças e agravos. A Educação Interprofissional, nesse contexto, propõe que estudantes de graduação em saúde e profissionais inseridos nos serviços aprendam a cooperar de forma colaborativa na resolução de questões atreladas ao trabalho. Este objetiva relatar a vivência das práticas colaborativas desenvolvidas pelos bolsistas do Programa de Educação Interprofissional em Saúde acerca das ações de prevenção, promoção e educação relacionadas à saúde dos trabalhadores incorporados ao Sistema Único de Saúde. Estudo exploratório, descritivo, de abordagem quantitativa sobre o relato de experiência dos bolsistas no projeto Pet/Saúde Interprofissionalidade. A partir das ações de prevenção e promoção à saúde realizadas nas unidades de saúde, o sexo feminino foi o predominante; a formação de nível superior se fez presente na equipe; percebeu-se um alto índice de sobrepeso e obesidade nos trabalhadores que alegaram não receber capacitação frequentemente na unidade de atuação. Percebeu-se satisfação profissional no ambiente de trabalho. Relataram sofrer ocasionalmente e frequentemente dor na nuca, nas costas e na região lombar; o nível de dor relatado foi de desconfortável à totalmente horrível. A prática da reflexologia refletiu em uma diminuição no nível da dor entre os trabalhadores. Pode-se visualizar o trabalho como fator determinante do processo saúde-doença dos indivíduos e da coletividade. O cuidado à Saúde dos Trabalhadores almeja constituir um novo campo de atuação e intervenção da rede pública de serviços de saúde no Brasil. Palavras-chave: Saúde do trabalhador; Estresse ocupacional; Educação interprofissional; Práticas colaborativas.

\begin{abstract}
Occupational Health is characterized as a field of practices and strategic knowledge aimed at analyzing and intervening in work relationships that cause diseases and injuries. Interprofessional Education, in this context, proposes that undergraduate health students and professionals inserted in the services learn to cooperate collaboratively in the resolution of issues linked to work. This aims to report the experience of the collaborative practices developed by the scholarship holders of the Interprofessional Education Program in Health about prevention, promotion and education actions related to the health of workers incorporated into the Unified Health System. Exploratory, descriptive study, with a quantitative approach to the report experience of scholarship holders in the Pet / Health Interprofessionality project. From the prevention and health promotion actions carried out in the health units, the female gender was the predominant one; higher education was present in the team; a high rate of overweight and obesity was perceived in workers who claimed not to receive training frequently in the unit. Professional satisfaction was perceived in the work
\end{abstract}


environment. They reported occasionally and frequently suffering pain in the neck, back and lumbar region; the level of pain reported was from uncomfortable to totally horrible. The practice of reflexology reflected in a decrease in the level of pain among workers. Work can be seen as a determining factor in the health-disease process of individuals and the community. Care for Workers' Health aims to constitute a new field of action and intervention by the public health service network in Brazil.

Keywords: Occupational health; Occupational stress; Interprofessional education; Collaborative practices.

\section{Resumen}

La Salud Ocupacional se caracteriza por ser un campo de prácticas y conocimientos estratégicos orientados a analizar e intervenir en las relaciones laborales que provocan enfermedades y lesiones. La Educación Interprofesional, en este contexto, propone que los estudiantes de pregrado en salud y los profesionales insertados en los servicios aprendan a cooperar de manera colaborativa en la resolución de cuestiones vinculadas al trabajo. Este tiene como objetivo reportar la experiencia de las prácticas colaborativas desarrolladas por los becarios del Programa de Educación Interprofesional en Salud sobre acciones de prevención, promoción y educación relacionadas con la salud de los trabajadores incorporadas al Sistema Único de Salud. Estudio exploratorio, descriptivo, con un análisis cuantitativo Aproximación al informe experiencia de los becarios en el proyecto de Interprofesionalidad Mascota / Salud. De las acciones de prevención y promoción de la salud realizadas en las unidades de salud, el género femenino fue el predominante; la educación superior estuvo presente en el equipo; Se percibió una alta tasa de sobrepeso y obesidad en los trabajadores que afirmaron no recibir capacitación con frecuencia en la unidad. Se percibió satisfacción profesional en el entorno laboral. Informaron que ocasionalmente y con frecuencia padecían dolor en el cuello, la espalda y la región lumbar; el nivel de dolor informado fue de incómodo a totalmente horrible. La práctica de la reflexología se reflejó en una disminución del nivel de dolor entre los trabajadores. El trabajo puede verse como un factor determinante en el proceso salud-enfermedad de las personas y la comunidad. El cuidado de la salud de los trabajadores tiene como objetivo constituir un nuevo campo de acción e intervención de la red de servicios públicos de salud en Brasil.

Palabras clave: Salud ocupacional; Estrés laboral; Educación interprofesional; Prácticas colaborativas.

\section{Introdução}

A Saúde do Trabalhador é caracterizada como um campo de práticas e de conhecimentos estratégicos interdisciplinares, técnicos, sociais, políticos, humanos, multiprofissionais e interinstitucionais, voltados para analisar e intervir nas relações de trabalho que provocam doenças e agravos (Gomez et al, 2018).

A Saúde Coletiva é tida como seu marco referencial, ou seja, seu foco principal de atuação visa à promoção, à prevenção e à vigilância em saúde. $\mathrm{O}$ aspecto interdisciplinar se evidencia na tentativa de estabelecer e articular dois planos de análise, sendo um deles: o que contempla o aspecto social, econômico político e cultural, que por sua vez é definidor das relações particulares que ocorrem nos espaços de trabalho e da forma como os diferentes grupos humanos se reproduzem socialmente; e outro: o referente às características de processos de trabalho que apresentem potencial de repercussão na saúde (Gomez et al., 2018).

Uma experiência pioneira no Brasil, com efetiva participação sindical ocorreu em 1984 junto ao Sindicato dos Trabalhadores Químicos e Petroquímicos do ABCD, ao qual foi descrita pela Assessoria Técnica do Departamento Intersindical de Estudos e Pesquisas de Saúde e dos Ambientes de Trabalho - DIESAT, que relatou um forte aumento na organização dos trabalhadores em torno da regulamentação da jornada de trabalho e em busca de melhores salários naquela década (Lacaz, 2007).

Um dos marcos políticos normativos do Estado, na perspectiva da saúde, a Saúde do Trabalhador é considerada um direito universal, conforme está definido pela Constituição Federal de 1988 e na Lei nº 8080/90, vindo a transcender o limite do direito previdenciário-trabalhista em que a ação de Estado é restrita apenas à regulação da saúde e segurança (Gomez et al., 2018).

De acordo com dados provenientes de fontes oficiais, por todo o mundo persistem os acidentes e doenças com origem em processos de trabalho. Tanto dados nacionais quanto internacionais revelam a magnitude com que estes eventos afetam a Saúde Pública. A Organização Internacional do Trabalho (OIT) afirma que ocorrem por volta de 270 milhões acidentes de trabalho no mundo anualmente, sendo que 2 milhões têm consequências fatais. O Brasil encontra-se no terceiro lugar de acidentes 
fatais em números absolutos no ranking mundial, novamente atrás dos Estados Unidos e da China (Zinet, 2012; Costa, Lacaz \& Vilela, 2013; OIT, 2020).

Sabe-se, no entanto, que tais dados representam apenas uma parte do total dos acidentes efetivamente ocorridos, por excluírem agravos não registrados pelas empresas e os sofridos por trabalhadores do setor informal, que, segundo dados da Pesquisa Nacional por Amostra de Domicílios (PNAD), atingiram 37,4\% dos trabalhadores brasileiros em 2009 (Zinet, 2012).

A Educação interprofissional tem por objetivo promover que estudantes dos diferentes cursos de graduação em saúde e profissionais inseridos nos serviços aprendam a trabalhar juntos de forma colaborativa, ao permitir que cada profissão contribua com sua expertise (Peduzzi, 2016).

O trabalhador pode enfrentar consequências prejudiciais à sua saúde mental e física devido ao estresse contínuo ao qual é submetido durante sua jornada de trabalho. Como, por exemplo: desenvolver síndrome metabólica, sofrer distúrbios do sono, enfermidades psicossomáticas, síndrome de burnout, diabetes, hipertensão, depressão, absenteísmo, uso de substâncias psicoativas, além de queda na sua produtividade, surgimento e aumento de insatisfação laboral e redução na qualidade de vida no trabalho (Ribeiro et al., 2018).

Os profissionais de enfermagem e medicina passam por situações que os levam ao estresse, pelo fato de conviver em sua rotina com a dor do outro, o sofrimento e a morte, sendo submetidos a ritmos intensos de trabalho, longas jornadas, trabalho em turnos, salários insatisfatórios, relações humanas complexas, escassez de materiais e recursos humanos, e vários outros fatores que podem vir a desencadear estresse ou mesmo intensificá-lo (Ribeiro et al., 2018).

É de fundamental importância que a equipe do projeto PET/Saúde Interprofissionalidade desenvolvam atividades no setor de trabalho dos profissionais atuantes na unidade de saúde do SUS para que contribua com a melhora da qualidade de vida e a satisfação profissional e pessoal dos trabalhadores.

Diante disso, o objetivo deste artigo é relatar a vivência das práticas colaborativas desenvolvidas pelos bolsistas do Programa de Educação para o Trabalho Interprofissional acerca das ações de prevenção, promoção e educação relacionadas à saúde dos trabalhadores incorporados ao Sistema Único de saúde.

\section{Metodologia}

Trata-se de um relato de experiência, de natureza quantitativa, acerca da vivência das práticas colaborativas desenvolvidas pelos bolsistas do PET/Saúde Interprofissionalidade acerca das ações de prevenção, promoção e educação relacionadas à saúde dos trabalhadores desenvolvida no Centro Referencial de Saúde - Postão, no município de Cáceres, Mato Grosso (Pereira, 2014). O público-alvo contemplou uma parcela dos trabalhadores que atuavam na unidade de saúde mencionada. As atividades foram desenvolvidas durante 6 (seis) semanas, com um encontro semanal no período de outubro a dezembro do ano de 2019, as quais envolveram dinâmicas de grupo, medidas antropométricas e o controle da pressão arterial, bem como a disponibilização de um instrumento de avaliação para os participantes das atividades propostas.

A equipe do Programa PET-Saúde Interprofissionalidade da unidade foi composta por uma coordenadora local (Enfermeira Docente), um tutor (Fisioterapeuta Docente), duas preceptoras (Enfermeira e Farmacêutica), dois profissionais liberais (Psicólogo e Fisioterapeuta) e seis discentes, dentre eles: dois do curso de Medicina; dois de Enfermagem e dois de Educação Física, todos da Universidade do Estado de Mato Grosso - UNEMAT.

As atividades desenvolvidas na unidade de saúde seguiram a rotina de 3 etapas diárias: os discentes responsáveis pela atividade aferiram a pressão arterial dos trabalhadores e anotavam os valores das respectivas pressões arteriais. Posteriormente, os trabalhadores eram levados para a área externa da unidade, ao qual era realizado alongamentos e atividades de desenvolvimento cognitivo, com intuito de fortalecer a musculatura e melhorar a psicomotricidade do corpo. Ao término das 
atividades, conferiu-se a pressão arterial dos participantes novamente e anotado o valor, a fim de observar as variações da pressão arterial.

Os trabalhadores assinaram o Termo de Consentimento Livre e Esclarecido, após estarem cientes dos objetivos da pesquisa. Para a coleta de dados, os trabalhadores responderam o questionário autoexplicativo de áreas dolorosas, os questionários sociodemográficos, a Escala Visual Analógica (EVA), tanto antes, como após a intervenção da Reflexologia durante as atividades.

\section{Resultados}

As informações coletadas foram submetidas à codificação apropriada a partir da criação de um banco de dados. Foi utilizado o processo de validação dos dados por dupla digitação em planilhas do aplicativo Microsoft Excel. Os cálculos das análises estatísticas foram realizados com o auxílio do programa Statistical Package for Social Science (SPSS), versão 20.0 para cálculo das análises descritivas, de variabilidade (desvio padrão, máximo e mínimo), e de tendência central (média e mediana). As variáveis nominais, qualitativas, foram apresentadas com seus valores relativos e absolutos.

Foram 16 participantes que realizaram o preenchimento dos dados sociodemográficos, sendo 14 trabalhadores da unidade de saúde e 2 externos, 1 estudante e 1 docente. O perfil dos trabalhadores da Saúde do Centro de Referência em Saúde - Postão do município de Cáceres, dentre estes a maior parte foi do sexo feminino 86,7\% (n=13), sendo apenas 13,3\% (n=2) do sexo masculino. O estado civil dos participantes foi que 5 deles não possuem companheiros, o que corresponde a 33,3\% e, 9 possui companheiros (60\%) e 1 participante não respondeu à pergunta $(6,7 \%)$.

Sobre o grau de escolaridade, 4 dos participantes possuem o ensino médio completo (26,7\%), enquanto 8 dos participantes possuem curso superior completo (53,3\%), e 3 dos participantes possuem pós-graduação (20\%). Os resultados descritivos dos trabalhadores encontram-se na Tabela 1.

Tabela 1 - Distribuição dos participantes da pesquisa segundo características sociodemográfica e profissional. Cáceres - MT, Brasil, 2019.

\begin{tabular}{|c|c|c|}
\hline Dados Sociodemográficos & Média / desvio padrão & Mediana (Min. e Max.) \\
\hline Idade & $37,4 \pm 8,8$ & 35 (min. 21 e máx. 53) \\
\hline $\begin{array}{l}\text { Índice de Massa Corporal } \\
\text { (IMC) }\end{array}$ & $25,2 \pm 2,6$ & 25,3 (min. 21 e máx. 23) \\
\hline Escolaridade & $3,6 \pm 1,1$ & 4 (min. 2 e máx. 5) \\
\hline Número de pessoas em casa & $3,2 \pm 1,7$ & 3 (min. 1 e máx. 8) \\
\hline Número de cômodos em casa & $6,5 \pm 1,8$ & 7 (min. 3 e máx. 10) \\
\hline Número de filhos & $*$ & 1 (min. 0 e máx. 3) \\
\hline Renda & $4.844,33 \pm 4.647,11$ & $\begin{array}{c}3.000,00 \text { (min. } 998,0 \text { e máx. } \\
18.000,00)\end{array}$ \\
\hline Tempo de profissão & $8,1 \pm 7$ & 8,7 (min. 1 e máx. 22) \\
\hline Horas de trabalho por semana & $28,1 \pm 15,8$ & 28,2 (min. 6 máx. 40) \\
\hline $\begin{array}{l}\text { Total de horas de trabalho e } \\
\text { atividade domésticas }\end{array}$ & $55,2 \pm 37,2$ & 50 (min. 7 e máx. 133) \\
\hline
\end{tabular}

Legenda: * = média e desvio padrão não determinados. Fonte: Autores (2019). 
A Tabela 1 acima demonstra as principais características sociodemográficas dos profissionais trabalhadores como: o tempo de profissão; horas no trabalho e horas de trabalho somadas às atividades domésticas; a renda o número de filhos ou pessoas dentro da mesma casa, a idade e o índice de massa corporal. Estas características somadas ou isoladamente podem implicar diretamente na saúde física e mental dos trabalhados.

Tabela 2 - Distribuição dos participantes da pesquisa de acordo com o nível de IMC. Cáceres, MT, Brasil, 2019.

\begin{tabular}{ccc}
\hline Nível de IMC & Valor absoluto & Valor relativo $(\%)$ \\
\hline Adequado & 6 & 37,5 \\
Sobrepeso & 8 & 50 \\
Obeso & 2 & 12,5 \\
Total & 16 & 100 \\
\hline
\end{tabular}

Legenda: IMC = índice de massa corpórea. Fonte: Autores (2019).

Na Tabela 2 acima, pode-se observar que a maioria dos participantes 50\% encontra-se em sobrepeso e dois participantes encontram-se obesos.

Realizou-se o levantamento das áreas de atuação dos trabalhadores da unidade, conforme a Tabela 3.

Tabela 3 - Distribuição dos participantes da pesquisa de acordo com o setor de trabalho na unidade de saúde e/ou profissão. Cáceres - MT, Brasil, 2019.

\begin{tabular}{ccc}
\hline Setor de trabalho e/ ou Profissão & Valor absoluto & Valor relativo (\%) \\
\hline Agente Comunitário de Saúde & 3 & 20 \\
Assistente Social & 1 & 6,7 \\
Auxiliar de Enfermagem & 1 & 6,7 \\
Administrativo & 2 & 13,3 \\
Farmacêutico & 1 & 6,7 \\
Auxiliar Odontológico & 1 & 6,7 \\
Auxiliar de Limpeza & 1 & 6,7 \\
Estudante & 1 & 6,7 \\
Docente & 1 & 6,7 \\
Estagiário & 3 & 20 \\
Total & 15 & 100 \\
\hline
\end{tabular}

Fonte: Autores (2019).

Conforme a Tabela 3 acima o setor/profissão mais frequentes dos trabalhadores foram indicados, dentre estes os mais frequentes foram Agente comunitário de saúde e Estagiário.

Os trabalhadores foram questionados se possuíam algum tipo de capacitação na unidade, seis trabalhadores responderam que não possuíam nenhum tipo de capacitação $(26,1 \%)$, enquanto nove dos participantes $(39,1 \%)$ responderam que possuíam capacitação no atual emprego. 
Os trabalhadores foram investigados sobre o nível de satisfação no trabalho, conforme a Tabela 4.

Tabela 4 - Distribuição dos participantes da pesquisa de acordo com o nível de satisfação dos trabalhadores da unidade. Cáceres - MT, Brasil, 2019.

\begin{tabular}{ccc}
\hline Nível de Satisfação no trabalho & Valor absoluto & Valor relativo (\%) \\
\hline Muito insatisfeito & 1 & 6,7 \\
Insatisfeito & 2 & 13,3 \\
Neutro & 3 & 20 \\
Satisfeito & 6 & 40 \\
Muito Satisfeito & 2 & 13,3 \\
Total & 14 & 93,3 \\
Não respondeu & 1 & 6,7 \\
Total & 15 & 100 \\
\hline
\end{tabular}

Fonte: Autores (2019).

Conforme a Tabela 4 acima, quando é indagado em relação ao nível de satisfação com as tarefas desempenhas no trabalho a maioria dos participantes $40 \%$ sentem-se satisfeitos, $20 \%$ Não sabe (Neutro) e 13,30\% estariam insatisfeitos e muito satisfeitos. Apenas um participante mostrou insatisfação total com as tarefas desempenhadas.

Investigou-se o nível da dor (Gráfico 1), a frequência das áreas dolorosas (Tabela 4) que mais afetam os trabalhadores do Centro de Referencial em Saúde. Ao investigar as áreas dolorosas e a frequência da dor entre os trabalhadores, observou-se que 56,3\% dos participantes possuem dor na nuca ocasionalmente e frequentemente. As dores nas costas apresentadas pelos trabalhadores como ocasionalmente e frequentemente foi de 50\%. Enquanto a manifestação da dor na região lombar foi de $56,3 \%$, conforme a Tabela 5 .

Tabela 5 - Distribuição das áreas dolorosas citadas pelos trabalhadores da Saúde do Centro de Referência em Saúde do município de Cáceres obtidos por meio do questionário de áreas dolorosas, Cáceres, MT, Brasil, 2019.

\begin{tabular}{|c|c|c|c|c|}
\hline Área dolorosa/ Frequência & $\begin{array}{l}\text { Média / desvio } \\
\text { padrão }\end{array}$ & Mediana & $\begin{array}{c}\text { Valor } \\
\text { absoluto }\end{array}$ & Valor relativo $(\%)$ \\
\hline \multicolumn{5}{|l|}{ Nuca } \\
\hline -Ocasionalmente & & & 3 & 18,8 \\
\hline -Frequentemente & $2,25 \pm 0,77$ & 2 & 6 & 37,5 \\
\hline -Sem dor & & & 7 & 43,8 \\
\hline -Total & & & 16 & 100 \\
\hline \multicolumn{5}{|l|}{ Cotovelo } \\
\hline -Ocasionalmente & & & 3 & 18,8 \\
\hline -Frequentemente & $2,56 \pm 0,81$ & 3 & 1 & 6,3 \\
\hline -Sem dor & & & 12 & 75 \\
\hline -Total & & & 16 & 100 \\
\hline \multicolumn{5}{|l|}{ Antebraco } \\
\hline -Ocasionalmente & & & 1 & 6,3 \\
\hline -Frequentemente & $2,81 \pm 0,54$ & 3 & 1 & 6,3 \\
\hline -Sem dor & & & 14 & 87,5 \\
\hline -Total & & & 16 & 100 \\
\hline
\end{tabular}


Research, Society and Development, v. 10, n. 3, e50710313589, 2021

(CC BY 4.0) | ISSN 2525-3409 | DOI: http://dx.doi.org/10.33448/rsd-v10i3.13589

\begin{tabular}{|c|c|c|c|c|}
\hline \multicolumn{5}{|l|}{ Pulso/mão } \\
\hline -Ocasionalmente & & & 2 & 12,5 \\
\hline -Frequentemente & $2,69 \pm 0,70$ & 3 & 1 & 6,3 \\
\hline -Sem dor & & & 13 & 81,3 \\
\hline -Total & & & 16 & 100 \\
\hline \multicolumn{5}{|l|}{ Coxa } \\
\hline -Ocasionalmente & & & 4 & 25 \\
\hline -Frequentemente & $2,44 \pm 0,89$ & 3 & 1 & 6,3 \\
\hline -Sem dor & & & 11 & 68,8 \\
\hline -Total & & & 16 & 100 \\
\hline \multicolumn{5}{|l|}{ Panturrilha } \\
\hline Ocasionalmente & & & 4 & 25 \\
\hline -Frequentemente & $2,44 \pm 0,89$ & 3 & 1 & 6,3 \\
\hline -Sem dor & & & 11 & 68,8 \\
\hline -Total & & & 16 & 100 \\
\hline \multicolumn{5}{|l|}{ Ombro } \\
\hline -Ocasionalmente & & & 3 & 18,8 \\
\hline -Frequentemente & $2,37 \pm 0,80$ & 3 & 4 & 25 \\
\hline -Sem dor & & & 9 & 56,3 \\
\hline -Total & & & 16 & 100 \\
\hline \multicolumn{5}{|l|}{ Costa } \\
\hline -Ocasionalmente & & & 4 & 25 \\
\hline -Frequentemente & $2,25 \pm 0,85$ & 2,5 & 4 & 25 \\
\hline -Sem dor & & & 8 & 50 \\
\hline -Total & & & 16 & 100 \\
\hline \multicolumn{5}{|l|}{ Lombar } \\
\hline -Ocasionalmente & & & 3 & 18,8 \\
\hline -Frequentemente & $2,25 \pm 0,77$ & 2 & 6 & 37,5 \\
\hline -Sem dor & & & 7 & 43,8 \\
\hline -Total & & & 16 & 100 \\
\hline \multicolumn{5}{|l|}{ Quadril } \\
\hline -Ocasionalmente & & & 4 & 25 \\
\hline -Frequentemente & $2,37 \pm 0,88$ & 3 & 2 & 12,5 \\
\hline -Sem dor & & & 10 & 62,5 \\
\hline -Total & & & 16 & 100 \\
\hline \multicolumn{5}{|l|}{ Joelhos } \\
\hline -Ocasionalmente & & & 3 & 18,8 \\
\hline -Frequentemente & $2,5 \pm 0,81$ & 3 & 2 & 12,5 \\
\hline -Sem dor & & & 11 & 68,8 \\
\hline -Total & & & 16 & 100 \\
\hline \multicolumn{5}{|l|}{ Tornozelos/pé } \\
\hline -Ocasionalmente & & & 2 & 12,5 \\
\hline -Frequentemente & $2,69 \pm 0,70$ & 3 & 1 & 6,3 \\
\hline -Sem dor & & & 13 & 81,3 \\
\hline -Total & & & 16 & 100 \\
\hline
\end{tabular}

Fonte: Autores (2019).

Conforme a Tabela 5 acima, as áreas dolorosas do corpo dos trabalhadores foram investigadas por região (Nuca, cotovelo, antebraço, pulso/mão, coxa, panturrilha, costas, lombar, quadril, joelhos e tornozelos/pés) e por frequência do aparecimento da dor (ocasionalmente, frequentemente ou sem dor). Dentre tais, as áreas da nuca e lombar foram as mais relatadas com dor que se apresenta com frequência em 37,5\% (ambas as áreas lombar e nuca). A área sem dor mais relatadas pelos trabalhadores foi o antebraço $(87,5 \%)$. 
Foi investigado o nível de dor e constatou-se que a resposta de desconfortável à totalmente horrível entre os trabalhadores foi de $41,6 \%$, conforme Gráfico 1 , antes dos participantes terem feito a intervenção com a prática da reflexologia. Entretanto, observou-se que os participantes que tinham o nível de dor totalmente horrível representado por 8,3\% dos participantes, obtiveram melhoras após a intervenção da reflexologia, obtendo 75\% dos participantes sem dor.

Gráfico 1 - Distribuição de relatos de dor de trabalhadores da Saúde do Centro de Referência em Saúde do município de Cáceres obtidos por meio da Escala Visual Analógica (EVA) antes da intervenção (reflexologia) Cáceres, MT, Brasil, 2019.

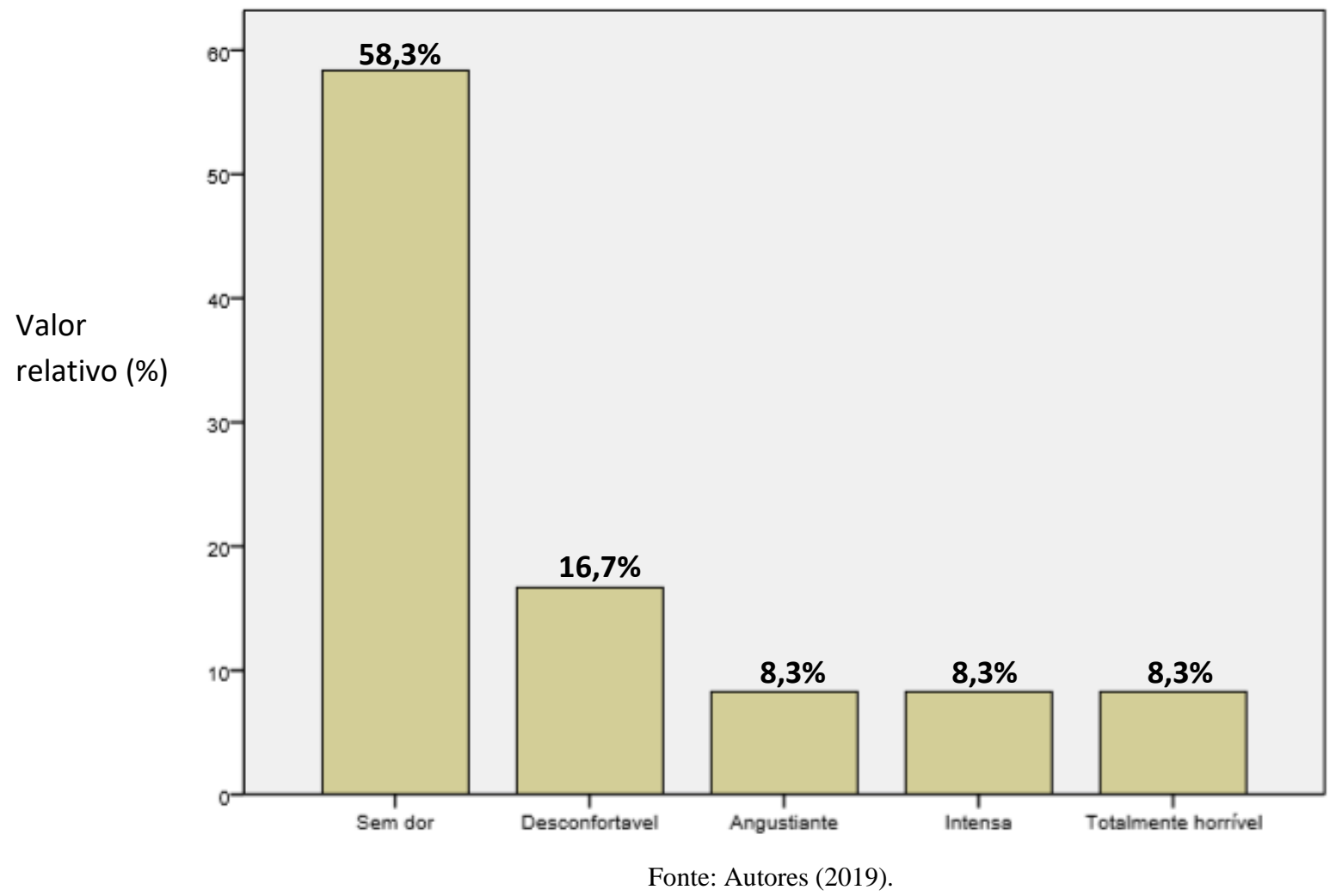

Conforme o Gráfico 1 acima pode-se perceber que antes da intervenção de reflexologia 58,3\% dos trabalhadores estavam sem dor. Dentre os trabalhadores que estavam com dor ao se analisar o nível de dor a maioria $(16,7 \%)$ relata tal sensação como desconfortável. O restante dos participantes com dor apresenta um nível de dor maior que vai de angustiante a totalmente horrível.

Antes da intervenção da reflexologia os trabalhadores sem dor eram representados por 58,3\% e com dor desconfortável eram de $16,7 \%$, o qual diminuiu após intervenção para $8,3 \%$, ou seja, demonstrado melhora significativa do nível da dor após a intervenção de reflexologia entre os participantes, conforme os Gráficos 1 e 2. 
Gráfico 2 - O nível da dor dos trabalhadores do Centro Referência em Saúde do município de Cáceres obtidos por meio da EVA após a intervenção.

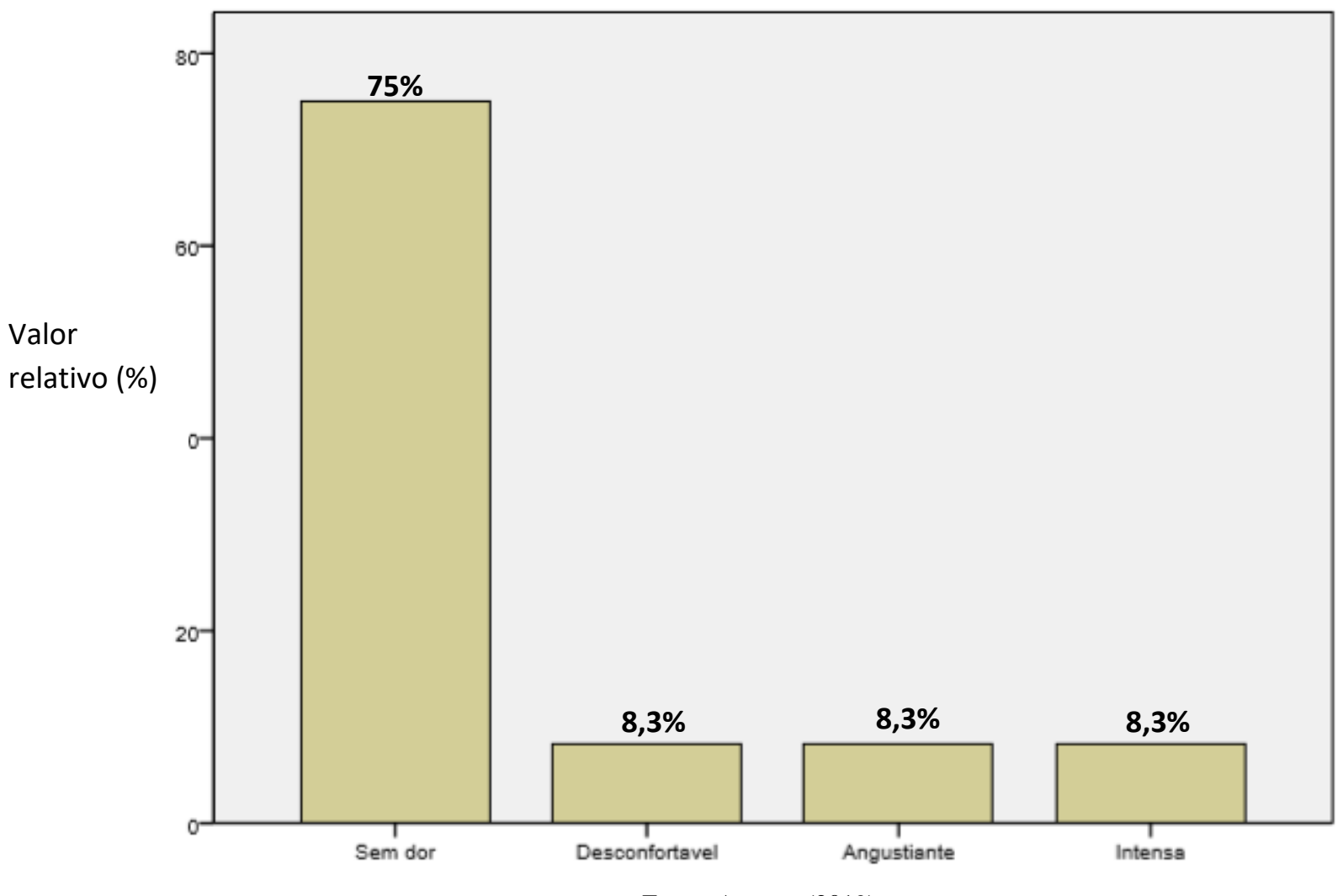

Fonte: Autores (2019).

Conforme o Gráfico 2 acima, após a intervenção da prática da reflexologia, percebeu-se a diminuição no nível da dor entre os participantes: em desconfortável no gráfico 1 antes da reflexologia o valor foi de 16,7 e após a reflexologia (Gráfico 2) 8,3\%. Embora os níveis de dor angustiante e intensa permaneceram inalterados após a técnica de reflexologia (Gráfico 2), percebe-se que o nível “totalmente horrível” não é mais relatado por nenhum participante no Gráfico 2.

\section{Discussão}

O trabalho pode participar de três formas no desenvolvimento de doenças: como uma causa necessária; como um fator contributivo, mas não necessário; e como provocador de um distúrbio latente ou agravador de uma doença já estabelecida, como uma causa (Dias, 2001).

A maior parte dos participantes do presente estudo (62,5\%) apresentavam fatores de risco como a obesidade e o sobrepeso, semelhante aos resultados encontrados em outro estudo que também analisou trabalhadores da área da saúde (64\% dos participantes) (Souza et al., 2012).

Segundo Andrade (2001), trabalhadores que permanecem sentados durante a maior parte da jornada de trabalho apresenta um estilo de vida sedentário e relevante fator de risco à saúde como a obesidade que favorece doenças metabólicas. No presente estudo, apesar da obesidade/ sobrepeso ter sido vistas na maior parte dos participantes, a área de atuação da maioria dos indivíduos (20\% de Agentes comunitários e estagiários) não justifica o maior tempo sentado para favorecer tal quadro de sedentarismo e possível risco de obesidade.

Alterações nas estruturas musculares e esqueléticas representam um grave problema para o indivíduo e, mesmo de saúde pública, uma vez que consta de uma das mais importantes causas de incapacidades e ausência do trabalhador no ambiente de trabalho (Barreto, 2001). 
De acordo com Zapater et al. (2004), fatores como o aumento da pressão interna no núcleo do disco intervertebral, estiramento dos ligamentos, pequenas articulações e nervos, geram alterações nas estruturas músculos-esqueléticas da coluna lombar, e geram uma redução na circulação de retorno dos membros inferiores promovendo desconfortos na região do pescoço e membros superiores.

É sabido que a permanência dos indivíduos em posturas incorretas por longos períodos é capaz de gerar desequilíbrios na musculatura, os quais possibilitam um ciclo vicioso de má postura e consequentemente dor nas costas (Toscano \& Egypto, 2001).

Sabe-se que a Qualidade de Vida no Trabalho influencia diretamente na Qualidade de Vida Total, uma vez que convivemos no ambiente de trabalho mais de oito horas por dia, durante pelo menos 35 anos durante a vida (Ramos et al., 2014). É fundamental a implementação de melhorias no ambiente de trabalho. É válido mencionar que o tempo de atividades de trabalho é muitas vezes somado às atividades domésticas (Ramos et al., 2011; Song, \& Baicker, 2019).

O uso da ginástica laboral, a qual promoveu melhoria da integração entre os funcionários, auxiliou no combate ao estresse e às lesões do dia a dia e ainda o incremento da flexibilidade (Salve \& Teodoro, 2004). A utilização de um programa de atividade física com exercícios de alongamento muscular e/ou associados a capacidades como resistência aeróbica, força e atividades de lazer, tem sido um dos recursos importantes que algumas empresas têm adotado, resultando em melhorias para a instituição e para ao trabalhador (Salve \& Teodoro, 2004; Cordes at al., 2019).

Tendo em vista os resultados obtidos por meio da técnica de reflexologia neste estudo, poder-se-ia propor o uso desta ou de outra técnica para a melhoria da qualidade de vida dos trabalhadores (Järnefelt et al., 2020).

\section{Conclusão}

A saúde do trabalhador é um campo que necessita de mais atenção frente aos problemas enfrentados no campo de atuação e práticas laborais, especialmente aos que desenvolvem atividades na área da saúde. Assim, este estudo desenvolvido ao longo das ações do PET/Saúde Interprofissionalidade, identificou fatores que contribuem para a prevenção, promoção e educação em saúde dos colaboradores do Centro Referencial de Saúde - Postão.

Em relação ao perfil sociodemográfico, identificamos que existe uma predominância de mulheres no campo de atuação da unidade, grande parte dos sujeitos possui nível superior completo.

Os principais problemas que acometem os trabalhadores são dores, sendo de maior incidência na região da nuca, costas e na região lombar. Observando estes empasses enfrentados pelos trabalhadores da unidade, foi ofertada uma atividade de educação sobre a saúde do trabalhador, e reflexologia aos indivíduos supracitados, por fim observou-se melhora após a atividade desenvolvida, deixando clara a necessidade de desenvolver no cotidiano dos colaboradores ações que contribuem para a melhora no estado físico e mental do trabalhador da unidade básica de saúde.

Por conseguinte, compreende-se visualizar o trabalho como fator determinante do processo saúde-doença dos indivíduos e da coletividade, o cuidado à saúde dos trabalhadores almeja constituir um novo campo de atuação e intervenção da rede pública de serviços de saúde no Brasil.

Sugere-se também que, com o avanço das pesquisas e a descoberta de novas informações acerca dessa temática, sejam realizados novos estudos a fim de sintetizar e divulgar soluções práticas e adequadas para a melhoria da saúde do trabalhador.

\section{Referências}

Andrade, A. (2001). Ocorrência e controle subjetivo do stress na percepção de bancários ativos e sedentários: a importância do sujeito na relação atividade física e saúde. 
Costa, D., Lacaz, F. A.C., \& Jackson, J. M. F . (2013). Saúde do Trabalhador no SUS: desafios para uma política pública. Revista brasileira de saúde ocupacional, 38(127).

Cordes, T., Bischoff, L. L., Schoene, D., Schott, N., Voelcker-Rehage, C., Meixner, C., Appelles, L. M., Bebenek, M., Berwinkel, A., Hildebrand, C., Jöllenbeck, T., Johnen, B., Kemmler, W., Klotzbier, T., Korbus, H., Rudisch, J., Vogt, L., Weigelt, M., Wittelsberger, R., Zwingmann, K., ... Wollesen, B. (2019). A multicomponent exercise intervention to improve physical functioning, cognition and psychosocial well-being in elderly nursing home residents: a study protocol of a randomized controlled trial in the PROCARE (prevention and occupational health in long-term care) project. BMC geriatrics, $19(1)$, 369.

Dias, E. C. (2001). Doenças relacionadas ao trabalho: Manual de procedimentos para os serviços de saúde. Ministério da Saúde do Brasil.

Gomes, M. C. Vasconcellos, L. C. F., \& Machado, J. M. H. (2018). Saúde do trabalhador: aspectos históricos, avanços e desafios no Sistema Único de Saúde. Ciência \& Saúde Coletiva, 23(6), 1963-1970.

Järnefelt, H., Härmä, M., Sallinen, M., Virkkala, J., Paajanen, T., Martimo, K. P., \& Hublin, C. (2020). Cognitive behavioural therapy interventions for insomnia among shift workers: RCT in an occupational health setting. International archives of occupational and environmental health, 93(5), 535-550.

Lacaz, F. A. C. (2007). O campo Saúde do Trabalhador: resgatando conhecimentos e práticas sobre as relações trabalho saúde. Caderno Saúde Pública, 23(4), 757-766.

Organização Internacional do Trabalho - OIT. (2020). Banco de dados ILOSTAT [banco de dados]. https://ilostat.ilo.org/data/.

Peduzzi, M. (2016). O SUS é interprofissional. Interface - Comunicação Saúde Educação, 20(56), 199-201.

Pereira, M. G. (2014). Artigos científicos: como redigir, publicar e avaliar. Rio de Janeiro: Guanabara Koogan.

Ramos, E. L., Souza, N. O. D. V. Gonçalves, F. A. G., Pires, A. S., \& Santos, D. M. (2014). Qualidade de vida no trabalho: repercussões para a saúde do trabalhador de enfermagem de terapia intensiva. Revista de Pesquisa Cuidado é Fundamental Online, 5(2), 571-583.

Ribeiro, R. P., Marziale, M. H. P., Martins, J. T., Galdino, M. J. Q., \& Ribeiro, P. H. V. (2018). Estresse ocupacional entre trabalhadores de saúde de um hospital universitário. Revista Gaúcha de Enfermagem, 39, 1-6.

Salve, M. G. C., \& Theodoro, P. F. R. (2004). Saúde do trabalhador: a relação entre ergonomia, atividade física e qualidade de vida. Salusvita, 23(1), 137-146.

Santos, B. S. F., \& Barreto, S. M. (2001). Atividade ocupacional e prevalência de dor osteomuscular em cirurgiões-dentistas de Belo Horizonte. Caderno Saúde Pública. 17(1), 181-193.

Song, Z., \& Baicker, K. (2019). Effect of a Workplace Wellness Program on Employee Health and Economic Outcomes: A Randomized Clinical Trial. JAMA, 321(15), 1491-1501.

Souza, C.A., Wittkopf, P. G., \& Thofehrn, C. (2012). Saúde do trabalhador: cuidados da saúde ocupacional dos servidores da área hospitalar. HU Revista, 37(3), $325-329$.

Toscano J., \& Egypto, E. (2001). A influência do sedentarismo na prevalência de lombalgia. Revista Brasileira de Medicina do Esporte, 7(4), $132-137$.

Zapater, A. R., Silveira, D. M., Vitta, A., Padovani, C. R., \& Silva, J. P. C. (2004). Postura sentada: a eficácia de um programa de educação para escolares. Ciência\& Saúde Coletiva, 9(1), 191-199.

Zinet, C. (2012). Condições pioram, acidentes aumentam: número de acidentes de trabalho aumenta na última década, preocupa sindicatos e organismos internacionais, que culpam a forma de produção. Caros Amigos, 187, 16-19. 